\title{
Evaluation of an alcohol screening and brief advice training programme for NHS general dental practitioners
}

A. Ntouva ${ }^{1}$, R. Sanatinia ${ }^{2}$ and R. G. Watt ${ }^{1}$

1 Department of Epidemiology and Public Health, University College London, London, UK,

2 Centre for Mental Health, Imperial College London, London, UK

\section{Keywords}

alcohol brief advice; dental education; general dental practice.

\section{Correspondence}

Antiopi Ntouva

Department of Epidemiology and Public Health, University College London, London, UK

Tel: 02076791703

Fax: 02078130280

e-mail: a.ntouva@ucl.ac.uk 


\section{Abstract}

Introduction: Primary care dentists are in a unique position to screen for alcohol misuse and provide brief advice to patients; however, lack of knowledge and confidence are some of the barriers cited for their reluctance to do so.

Aims: This study describes the evaluation of a novel brief alcohol advice training programme specifically designed and tailored to the needs of NHS general dental practitioners.

Materials and Methods: The training programme was developed as part of a feasibility trial and included a mixture of theoretical and practical elements. During $8 \mathrm{~h}$ of highly interactive sessions, participants took part in role-plays, including scenarios of patients of varying age groups, oral health status, dental attendance and different levels of motivation. Training manuals and other resources were also given. Knowledge, attitudes and confidence scores were assessed before and after the sessions. Wilcoxon matched-pairs signed-ranks and McNemar's tests were used to compare participants' performance before and after the completion of the programme.

Results: Significant positive changes were observed in all training objectives. There was an overall improvement of $23 \%$ in levels of knowledge, a $40 \%$ increase in positive attitudes towards alcohol screening and intervention and $80 \%$ increase in confidence scores. Fidelity assessments of the implementation of the intervention in the trial showed that dentists who completed the training successfully went on to screen for alcohol misuse and provide brief advice to patients.

Conclusion: NHS dentists can be successfully trained with the relevant knowledge, attitudes and confidence needed to deliver alcohol screening and brief advice to patients. 


\section{Introduction}

Alcohol misuse is a significant public health problem with major health, social and economic consequences (1). NICE has highlighted the need for both population measures and individual interventions to reduce alcohol harm (2). The majority of alcohol problems are not due to individuals with significant alcohol dependence, but rather to the much larger group of hazardous and harmful drinkers (3). The greatest impact can therefore be achieved by reducing alcohol intake amongst those drinking at increasing and higher risk levels. Primary care provides a useful setting for the early detection and provision of brief advice on alcohol misuse to the population (4). Whilst it is primarily general practitioners who have taken up the provision of alcohol brief advice in primary care, screening and advice are not performed systematically and rigorously. Data from The Health Improvement Network (THIN) primary care database looking at adult patients who registered at general practices between 2007 and 2009 found that 76\% (292,376 patients) had an entry regarding alcohol consumption, but only $9 \%$ were recorded to have completed a validated alcohol screening tool in the year after registration (5). Moreover, data using cross-sectional household surveys of representative samples

of adult populations in England, conducted monthly between March 2014 and November 2014 as surveys part of the ongoing Smoking Toolkit Study and Alcohol Toolkit Study, showed of the 1110 patients who were drinking above the recommended levels, $6.5 \%$ recalled being given advice by their GP, compared to $50.4 \%$ of 1775 smokers (6). This gap in the provision of validated screening and systematic delivery of brief advice in primary care provides an additional opportunity for other primary care professionals to pick up patients who are drinking excessively and may have been missed. General dental practice has therefore been identified as a potentially ideal setting to screen for alcohol misuse and provide brief advice (7). 
Despite the links of alcohol misuse to oral diseases such as increased risk of oral cancer (8), tooth surface loss (9), dental trauma (10), tooth staining and halitosis $(11,12)$ which make the issue of alcohol misuse directly relevant to dental professionals and their patients, dentists are reluctant to screen for alcohol misuse and very rarely provide alcohol brief advice to their patients (13-15). However, the 23,000 NHS dentists in the UK could play a major role in reducing alcohol consumption across the general population. Nearly $60 \%$ of adults ( 29.8 million) were seen by an NHS dentist over a 2-year period (16). Regular dental attendance is particularly high amongst women with nearly $70 \%$ reporting to be regular users of NHS dentists (17). Dentists therefore have regular contact with a significant segment of the 'healthy' adult population, many of whom do not have contact with other health professionals. Increasingly, dentists are more involved in providing preventive advice. For example, most NHS dentists now ask their patients about their diet and tobacco use and provide advice as required (18).

The main barriers limiting dentists' involvement in providing alcohol advice include lack of knowledge and training on alcohol, limited confidence in giving advice, and fear that the relationship with their patients would be damaged if they raised the potentially sensitive topic of alcohol use with their patients $(19,20)$. These barriers have also been identified in other settings, most widely in primary care where the majority of the interventions take place (21).

Brief alcohol advice training programmes have been developed for other health professionals. For example in the Screening and Intervention Programme for Sensible drinking (SIPS) in primary care settings, primary care staff received an hour of training on how to deliver 5 min of brief advice. The training was delivered by alcohol health workers in small groups of clinicians $(22,23)$. Similarly, a short training session was provided to clinicians in sexual health clinics for the Sexual Health and Excessive Alcohol Randomised trial (SHEAR) using recommended text in delivering the components of the intervention, as well as resources from the alcohol learning centre (24). To our knowledge, no alcohol 
screening and brief advice training programme specifically designed for NHS dentists has been developed and comprehensively evaluated.

The aim of this study was to describe the evaluation of a novel brief alcohol advice training programme specifically designed and tailored to the needs of NHS general dental practitioners.

\section{Materials and methods}

Ethical approval for the study was obtained from the Camden and Islington Research Ethics Committee (Reference: 13/LO/0292, Protocol V.3, 12/03/2014). Research and development approval was also obtained from Islington, Camden, Redbridge, Haringey, Enfield, Barnet and Brent former Primary Care Trusts, respectively. The training programme was part of a feasibility randomised controlled trial (Dental Alcohol Reduction Trial - DART) which aimed to assess the acceptability and practicality of an alcohol brief advice intervention delivered in primary dental settings across North London, UK. A more detailed overview of feasibility study methodology has been published elsewhere (25).

\section{Programme design}

The training programme was designed with assistance from an experienced alcohol intervention trainer and was based on previous training programmes targeted at other health professionals $(24,26,27)$. In addition, information gathered from separate focus groups with dental professionals and dental patients was used to tailor the programme specifically to meet the needs of NHS dentists working in primary care settings. Figure 1 describes the different elements of the training sessions.

The training was structured over two half-day sessions (a total of $8 \mathrm{~h}$ ). It comprised short theoretical inputs followed by highly interactive sessions including exercises and role-plays of increasing complexity. The theory sessions aimed at building the practitioners' knowledge on alcohol epidemiology, its impacts on the society, economy and health and in particular oral health. These sessions were brief and 
contained information that dentists were likely to be asked by patients, making them directly relevant to the participants. Concise key messages after each session were included in bullet point form to consolidate the key points to remember. Another important aspect of the theoretical training included training on units of alcohol and risk levels of drinking. Exercises in units of alcohol ensured that dentists had a clear idea of units in popular drinks and knew how to explain the concept to their patients in a clear way. Dentists were also provided with unit calculator ready reckoners to have fast access to information on units about all types of alcoholic drinks. Risk levels of drinking were also an important element as it was imperative that the dental professionals acknowledged what each risk level meant and how this would be communicated to the patient.

Before progressing on to the more practical elements of the training session, the attitudes of dental professionals towards alcohol brief advice in their practice were explored and discussed. Potential barriers were expressed, and the trainers outlined how the training programme will address these. The practical sessions included a variety of different methods. The first set of exercises aimed at building the participants' confidence in using the knowledge acquired from the previous sessions. The AUDIT-C screening tool was introduced along with sample scripts on how to introduce it to patients and how to provide feedback on the scores. The first set of AUDIT-C exercises involved completing the tool in several scenarios (different age groups, varying levels of oral health status and dental attendance) and deciding the action to be taken based on the score. As participants got more confident using the tool, a series of role-plays were introduced whereby the participants had to raise the issue of alcohol, go through the AUDIT-C tool and provide feedback based on the score. The role-plays went a step further by asking participants to assess the patient's motivation to change, in order to decide how they would proceed to providing brief advice. As previously mentioned, the role-plays included a range of different age groups, with varying socioeconomic circumstances and backgrounds. 
Similarly, the tailored brief advice tool was broken down into its individual elements and each one was described along with suggested scripts. A video showing a GP providing brief advice to a patient was also shown at this stage, to give participants a complete picture of how the advice is delivered in a clinical setting. The practical sessions for this part of the training were split into two groups. First, participants had to provide brief advice to a variety of patients who were compliant and amenable to change. This way the practitioners were given the opportunity to work through the practicalities of providing the advice and get more familiar with the brief advice tool. The participants were timed for this session to get an understanding of the time constraints of the intervention. After the end of the session, participants were asked to reflect on their performance as well as the performance of their colleague and discuss the difficulties in conducting the sessions, the techniques they used or their colleagues used that they can apply to their everyday practice. The final set of role-plays focused on more complex scenarios of patients less ready to comply with the advice. The focus of these role-plays was to assess the patient's motivation, address their concerns and decide which elements of the brief advice tool, if any, were appropriate to be given. In addition, local signposting information for patients with severe alcohol dependency was also covered during this session.

The consolidation phase involved reflections of the programme as a whole and a second look at the barriers initially cited by dentists and how the training programme managed to address them. Finally, a set of potential questions that the patients may have and possible answers were also provided. All participants received a training manual as well as resources for use in the practice.

Participants also received ongoing support by the research team after the training sessions, especially in the first few weeks of the brief advice implementation. They were encouraged to contact the team after the first few sessions in order to discuss any concerns and receive clarification if needed. 


\section{Programme evaluation}

At the beginning of the first session, participants were asked to complete a short questionnaire based on elements from the Short Alcohol and Alcohol Problems Perception Questionnaire which was used for the training questionnaires in SIPS (28). The questionnaire aimed to assess their baseline level of knowledge and attitudes in three key educational domains:

- Knowledge of alcohol and health: this section included eight questions which participants were asked to identify units of alcohol in popular drinks, current drinking guidelines in men and women, as well as other theoretical concepts such as the impact of alcohol on oral health and the principles of brief alcohol advice.

- Attitudes towards alcohol screening and intervention: this section included six attitudinal questions in which participants were asked to rate their level of agreement on a Likert scale of one (strongly disagree) to five (strongly agree) on a range of alcohol related topics.

- Confidence in delivering alcohol advice to patients: this section included four questions which participants were asked to rate their level of agreement on a scale of one (not very confident) to five (very confident) on different issues linked to providing alcohol screening and advice to dental patients.

At the end of the second day of training, the participants were asked to complete the same questionnaire. Some additional feedback questions were added to assess the perceived quality of the training sessions. Both pre- and post-training questionnaires were ID-coded so that the researchers were blinded to the responders' replies.

\section{Fidelity testing}

Dentists were advised to start delivering the brief advice intervention straight after the completion of the training programme in order to maintain momentum and gain confidence whilst the information 
was still recent in their minds. They were also asked to complete a fidelity form for each patient who received the brief intervention to ensure that the intervention was delivered consistently. The form was based on a checklist used in a previous trial (24), covered all components of the brief advice delivered and included a question asking how long it took to deliver the intervention.

\section{Statistical analysis}

Data were analysed using STATA 12 (29). Descriptive statistics (such as frequencies for categorical data and means, medians, standard deviations and interquartile ranges for numerical data) were used to analyse the sample characteristics at baseline. Cumulative scores were calculated for the three main training objectives (knowledge, attitudes and confidence) by aggregating scores for each question in each category. Each question from every objective was also explored separately. Wilcoxon matchedpairs signed-ranks tests were used to compare each participant's score before and after the completion of the training programme. For paired proportion differences, McNemar's test was used.

\section{Results}

\section{Sample characteristics}

Fifteen NHS dentists attended the training sessions. Fifty-seven per cent of the sample $(n=8)$ were male, and the mean age of the group was 37.6 (SD: 9.6). The sample included a mix of recently qualified graduates and dentists with extensive clinical experience (median years worked as a dentist: 12, IQR: 1, 22). The majority of the sample $(n=9,64 \%)$ had not attended any prior training on alcohol issues.

\section{Training outcomes}

There were significant improvements in all three training objectives. There was an overall improvement of $23 \%$ in levels of knowledge and more specifically significant improvements in identifying alcohol units 
in popular drinks and the impact of alcohol on oral health (Table 1). There was a $40 \%$ overall increase in positive attitudes towards alcohol screening and intervention, with the highest changes towards advising patients on the effect of alcohol and oral health, defining units and referring dependent drinkers (Table 2). Finally, confidence scores were also greatly increased by $80 \%$ overall, with dental professionals feeling significantly more confident by the end of the training sessions in discussing the negative impact of alcohol with their patients, screening patients for risky drinking using the AUDIT-C tool and subsequently providing brief advice. Detailed scores and breakdown of each objective are presented in the tables below.

\section{Feedback on training programme}

All participants agreed or strongly agreed that the course objectives were clearly defined, the delivery of the course was stimulating and interactive, that the knowledge gained would be useful in their practice, the resources were very useful and that they enjoyed the training. They also found the role-play exercises, the manual and all the training materials overall good or very good.

Fidelity of the intervention was assessed in 111 patients (93.3\%). All dentists who completed the training programme delivered successfully all the aspects of the intervention as assessed by the fidelity forms.

\section{Discussion}

This tailored training programme for NHS dentists achieved significant improvements in participants' knowledge, attitudes and confidence towards alcohol screening and provision of brief advice. Particularly impressive improvements occurred in the participants' attitudes and levels of confidence. To the best of our knowledge, no other studies have reported the evaluation of a training programme designed for practicing NHS dentists. Neff et al. (30) developed a 3-5 min motivational interviewing 
brief advice intervention for heavy drinkers delivered by dental hygienists. Analysis of audiotaped interventions showed that even though the fidelity to the protocol was high with regard to providing simple feedback, there was less compliance in the motivational interviewing elements. Miller and colleagues (31) designed and evaluated an online five-module programme on alcohol and oral health to undergraduate dental students and showed significant changes in their knowledge, attitudes and behaviours. Although the results of this student course were very encouraging, no information was gathered on whether the training had any long-term effects once the students had qualified as dentists.

It is important to recognise the limitations of our study. In particular, the modest sample size limits the generalisability of our results. As this was part of a feasibility study, the sample was restricted in size and only located in north London. It would have also been useful to conduct a longer term follow-up of the participants to examine whether the positive changes were maintained over a longer time period. In the feasibility study, we assessed the fidelity of the delivery of the training content through the use of an intervention checklist. This indicated high levels of compliance with the study protocol several months after the training had been delivered. An audio recording of the delivery of the brief advice and subsequent analysis by trained interventionalists would have provided a more objective assessment of the intervention fidelity. However, for the purposes of this feasibility study, this was thought to place additional pressure on practitioners and deter the participation of patients in the study based on consultations with patient and public involvement (PPI) members as well as clinicians. It was therefore agreed that a paper form would be more appropriate at this time and that an audio recording would be considered for a larger efficacy trial.

The strengths of this study include the comprehensive and well-designed training programme which included both theoretical and applied elements and that it was specifically designed for dentists based upon information gathered in separate exploratory focus groups with professionals and patients. 
Tailoring the programme to directly meet the educational and clinical needs of the dentists appears to have helped make it relevant and interesting to the participants.

\section{Conclusion}

This study has demonstrated that NHS dentists can be trained with the relevant knowledge, attitudes and confidence needed to deliver alcohol screening and brief advice to their patients. Future research will need to assess the effectiveness and cost effectiveness of dentists delivering brief alcohol advice.

\section{Funding}

This paper presents independent research funded by the National Institute for Health Research (NIHR) under its Research for Patient Benefit (RfPB) Programme (Grant Reference Number PB-PG-0212-27029). The views expressed are those of the author(s) and not necessarily those of the NHS, the NIHR or the Department of Health.

\section{Conflict of interest}

All authors declare there is no conflict of interest in relation to this study. 


\section{References}

1 Health and Social Care Information Centre. Statistics on Alcohol [Internet]. 2014. Available at: http://www.hscic.gov.uk/catalogue/PUB14184

2 NICE. Alcohol-use disorders: preventing the development of hazardous and harmful drinking. London: Public Health Guidance 24, 2010.

3 Drummond C, Adenekan O, Phillips T, et al. Alcohol needs assessment research project (ANARP). The national needs assessment for England. London: Department of Health and the National Treatment Agency, 2004.

4 O'Donnell A, Anderson P, Newbury-Birch D, et al. The impact of brief alcohol interventions in primary healthcare: a systematic review of reviews. Alcohol Alcohol 2014: 49: 66-78.

5 Khadjesari Z, Marston L, Petersen I, Nazareth I, Walters K. Alcohol consumption screening of newly registered patients in primary care: a cross-sectional analysis. Br J Gen Pract 2013: 63: e706-e712.

6 Brown J, West R, Angus C, et al. Comparison of brief interventions in primary care on smoking and excessive alcohol consumption: a population survey in England. Br J Gen Pract 2016: 66: e1-e9.

7 Shah NP, Goel R, McGurk M, Maroo A, Sproat C. Brief advice on alcohol: as easy as A..B..I? Br Dent J 2015: 218: 13-17. Nature Publishing Group.

8 Turati F, Garavello W, Tramacere I, et al. A meta-analysis of alcohol drinking and oral and pharyngeal cancers: results from subgroup analyses. Alcohol Alcohol 2013: 48: 107-118.

9 Robb ND, Smith BG. Prevalence of pathological tooth wear in patients with chronic alcoholism. Br Dent J 1990: 169: 367-369. 
10 Hutchison IL, Magennis P, Shepherd JP, Brown AE. The BAOMS United Kingdom Survey of Facial Injuries Part 1: aetiology and the association with alcohol consumption. Br J Oral Maxillofac Surg 1998: 36: 3-13.

11 Rosenberg M, Knaan T, Cohen D. Association among bad breath, body mass index, and alcohol intake. J Dent Res 2007: 86: 997-1000.

12 Suzuki N, Yoneda M, Naito T. The relationship between alcohol consumption and oral malodour. Int Dent J 2009: 59: 31-34.

13 Roked Z, Watson R, Moore S. Identification of alcohol misuse in dental patients. Fac Dent J 2014: 5: 134-137.

14 Shepherd S, Bonnetti D, Clarkson JE, Ogden GR, Young L. Current practices and intention to provide alcohol-related health advice in primary dental care. Br Dent J 2011: 211: E14. Nature Publishing Group, a division of Macmillan Publishers Limited. All Rights Reserved.

15 Paquette DW, Bell KP, Phillips C, Offenbacher S, Wilder RS. Dentists' knowledge and opinions of oralsystemic disease relationships: relevance to patient care and education. J Dent Educ 2015: 79: 626-635.

16 NHS England. Summary of the Dental Results from the GP Patient Survey; July to September 2014 [Internet]. 2014. Available at: https://www.england.nhs.uk/statistics/wp-content/uploads/sites/2/ 2015/01/GP-Patient-Survey-Dental-Results-Summary-1.pdf (accessed 2015 Nov 9)

17 Morris J, Chenery V, Douglas G, Treasure ET. Service considerations - a report from the Adult Dental Health Survey 2009. London: Health and Social Care Information Centre, 2011.

18 Yusuf H, Tsakos G, Ntouva A, et al. Differences by age and sex in general dental practitioners' knowledge, attitudes and behaviours in delivering prevention. Br Dent J 2015: 219: E7. 
19 Shepherd S, Young L, Clarkson JE, Bonetti D, Ogden GR. General dental practitioner views on providing alcohol related health advice; an exploratory study. Br Dent J 2010: 208: E13; discussion 3045. Nature Publishing Group.

20 McAuley A, Goodall CA, Ogden GR, Shepherd S, Cruikshank K. Delivering alcohol screening and alcohol brief interventions within general dental practice: rationale and overview of the evidence. $\mathrm{Br}$ Dent J 2011: 210: E15. Nature Publishing Group.

21 Johnson M, Jackson R, Guillaume L, Meier P, Goyder E. Barriers and facilitators to implementing screening and brief intervention for alcohol misuse: a systematic review of qualitative evidence. J Public Health (Oxf) 2011: 33: 412-421.

22 Kaner $\mathrm{E}$, Bland M, Cassidy $\mathrm{P}$, et al. Screening and brief interventions for hazardous and harmful alcohol use in primary care: a cluster randomised controlled trial protocol. BMC Public Health 2009: 9: 287.

23 Kaner EF, Dickinson HO, Beyer FR, et al. Effectiveness of brief alcohol interventions in primary care populations. Cochrane Database Syst Rev 2007: CD004148.

24 Crawford MJ, Sanatinia R, Barrett B, et al. The clinical and cost effectiveness of brief advice for excessive alcohol consumption among people attending sexual health clinics: a randomised controlled trial. Sex Transm Infect 2015: 91: 37-43.

25 Ntouva A, Porter J, Crawford MJ, et al. Assessing the feasibility of screening and providing brief advice for alcohol misuse in general dental practice: a clustered randomised control trial protocol for the DART study. BMJ Open 2015: 5: e008586.

26 Kaner E, Bland M, Cassidy P, et al. Effectiveness of screening and brief alcohol intervention in primary care (SIPS trial): pragmatic cluster randomised controlled trial. BMJ 2013: 346: e8501-e8501. 
27 NHS Health Scotland. Alcohol Brief Interventions training [Internet]. 2009. Available at: http://www.healthscotland.com/documents/5452.aspx

28 SIPS. Short Alcohol and Alcohol Problems Perception Questionnaire (SAAPPQ) Training questionnaires [Internet]. 2008. Available at: http://www.alcohollearningcentre.org.uk/Topics/Browse/BriefAdvice/

SIPS/BriefAdviceTrainingandTools/?parent $=4449 \&$ child $=4566$

29 StataCorp. Stata Statistical Software: Release 12. College Station, TX: StataCorp LP; 2011.

30 Neff JA, Walters ST, Braitman AL, et al. A brief motivational intervention for heavy alcohol use in dental practice settings: rationale and development. J Health Psychol 2013: 18: 542-553.

31 Miller PM, Ravenel MC, Mauldin MP, Sulkowski S, Lowndes A, Thomas SE. An online alcohol and oral health curriculum for dental students. J Dent Educ 2014: 78: 16-23. 